\title{
La conducta expectante y el seguimiento en las primeras semanas de vida de la displasia de cadera en el neonato previenen su sobretratamiento
}

Developmental dysplasia of the hip: a new approach to incidence.

Bialik V, Bialik GM, Shraga B et al. Pediatrics. 1999;103 (1):93-99.

\section{Objetivo}

Evaluar un algoritmo diagnóstico y terapéutico desarrollado anteriormente por los autores para identificar a aquellos neonatos con displasia de cadera, que de no ser tratada, desarrollarán alteraciones del desarrollo de la cadera (DDC).

Diseño

Seguimiento de una cohorte prospectiva.

Lugar

Neonatos nacidos en 1994 y 1995 que fueron sometidos a rastreo de DDC en el Ramban Medical Center de Haifa, Israel.

\section{Pacientes}

Se evaluó en forma consecutiva a 9030 neonatos (18 060 caderas). Fueron excluídos del estudio los neonatos con deformaciones esqueléticas, enfermedades neuromusculares o defectos del tubo neural. La población estuvo integrada por judíos israelitas, árabes, rusos y judíos egipcios.

\section{Descripción de los tests y del gold standard}

Desde 1992 este grupo de trabajo realizó rastreo ecográfico y clínico de más de 4000 neonatos, desarrollando a partir de las mismas, un algoritmo de manejo de la DDC en el neonato diseñado con el objetivo de prevenir el sobretratamiento de la DDC ${ }^{1}$.( Ver cuadro 1.)

Cuadro 1: Estrategia de manejo.

Todos los neonatos son examinados clínica y ecográficamente a la 24 horas y reexaminados clínicamente a las 72 horas:

1. Los neonatos sin factores de riesgo para DDC y sin hallazgos patológicos en el rastreo (clínico o ecográfico) son dados de alta.

2. Los que tienen la cadera estable al examen físico y algún factor de riesgo 0 inestabilidad ecográfica leve o moderada son reevaluados clínica y ecográficamente a las seis semanas; mientras que quienes presentan inestabilidad de la cadera al examen físico o inestabilidad ecográfica severa son reevaluados a las dos semanas.

Si la cadera en cuestión mejora son reevaludos con ambos métodos a las tres semanas y luego cada tres meses hasta el año de edad. Si no mejora o empeora se indica tratamiento, generalmente con el arnés de Pavlik.
Durante las primeras 48 horas los neonatos fueron examinados en forma independiente por dos observadores. El examen físico lo llevó a cabo un neonatólogo y el ecográfico, un cirujano traumatólogo. Las caderas fueron clasificadas en estables o inestables según el examen clínico (el estudio no especifica el método). Los hallazgos ecográficos fueron clasificados según la clasificación de Graf, que es la técnica ecográfica estática más difundida.

No se realizó seguimiento de los neonatos que fueron asumidos como "sanos" al nacimiento (ambas caderas normales).

\section{Medición de los resultados}

Según la evolución y de acuerdo a la estrategia mencionada ${ }^{1}$, se inició o nó el tratamiento. Aquellas caderas que no mostraron mejoría fueron tratadas, mientras que aquellas que mejoraron fueron controladas periódicamente hasta el año de vida, momento en el cual se las evaluó a través del examen físico, ecografía y radiología.

Los datos fueron reportados en número de caderas afectadas (en vez de neonatos) ya que solo una fracción de los niños afectados tuvo hallazgos bilaterales.

\section{Resultados principales}

A través del rastreo neonatal se detectaron 995 caderas con algún grado de anormalidad clínica y/o ecográfica. Esto significa una incidencia de 55 por cada 1000 nacidos. De acuerdo con el algoritmo seguido, solamente un 90 de estas 995 caderas necesitaron tratamiento, lo que lleva a una incidencia "real" de 5 de cada 1000 neonatos evaluados (este dato es interpretado por los autores como la verdadera incidencia de DDC). Las 905 caderas que fueron seguidas en forma sistemática pero que no fueron tratadas, permanecían normales al año de vida.

\section{Conclusiones}

La implementación de este algoritmo permite distinguir a los pacientes con DDC ecográfica de aquella que va a requerir tratamiento.

\section{COMENTARIO}

Existen discrepancias acerca de la utilidad de los métodos de rastreo empleados para la detección de DDC (hasta las maniobras de Barlowy Ortolani están incluidas en este debate). Esta controversia se relaciona con un pobre conocimiento de la historia natural de la enfermedad y con la falta de un criterios comunes de diagnóstico, lo que llevó a reportes de incidencias muy dispares (entre 0,04\% y el $51 \%$ ).

En los años sesenta Barlow describió que un $88 \%$ de los neonatos que presentaban inestabilidad de caderas al nacer se normalizaba sin tratamiento alguno a los dos meses ${ }^{2}$ y según algunas evaluaciones recientes, el rastreo neonatal por examen físico no habría logrado eliminar los casos de DDC de aparición tardía (Luego de los tres meses de edad) y que, generalmente, necesitan tratamiento quirúrgico. Un estudio Británico de vigilancia poblacional publicado recientemente reportó que la incidencia estimada de un primer procedimento quirúrgico para el tratamiento de DDC (indicador indirecto de la efectividad poblacional del programa nacional de rastreo) fue de 0.78 (IC95: 0.72-0.84) cada 1000 nacidos vivos, similar al que existía antes del programa y al de poblaciones no rastreadas ${ }^{3}$. Se piensa que esta dificultad en la detección se debería a una baja sensibilidad de las maniobras de rastreo, a falta de entrenamiento en quienes las realizan masivamente o bien, a una eficacia no total de los tratamientos no quirúrgicos. En los años ochenta se comenzó a discutir la utilidad de la ecografía como método alternativo y/o complementario del examen físico. Se observó que en un im-

\section{Referencias}

Dr. Juan Lucas Bernabó - Dr. Sergio Terrasa
Unidad de Medicina Familiar y Preventiva. Hospital Italiano de Buenos Aires. portante porcentaje las anormalidades detectadas ecográficamente eran aisladas ${ }^{4}$ (hallazgos ecográficos sin implicancia en el pronóstico clínico) y que si se definía a la DDC sólo ecográficamente, su incidencia aumentaba significativamente. Es de hacer notar que los autores reportan la incidencia de DDC considerando las "caderas" y no los pacientes examinados. Esto no es correcto desde el momento en que la probabilidad de displasia en una cadera no es independiente de la otra ya que los factores de riesgo asociados son los mismos (de hecho, el $20 \%$ son bilaterales). ${ }^{5}$

Los autores de este artículo proponen la existencia de dos entidades clínicas de pronóstico muy diferente: 1) una DDC que evoluciona a una cadera normal y que no significa más que un hallazgo ecográfico al nacer y 2) una verdadera DDC que va a progresar a algún grado de DDC. Evalúan un algoritmo de toma de decisiones diagnósticas y terapéuticas que habían desarrollado en un estudio anterior para diferenciar estas dos entidades. Esta estrategia previno que un $90,4 \%$ de aquellos pacientes que presentaban algún grado de anormalidad clínica o ecográfica al nacer sufrieran un tratamiento innecesario. Queda por determinar en estudios futuros llevados a cabo con mayor número de pacientes los factores predictores que identifiquen aquellos neonatos que desarollarán una verdadera DDC y si estas conclusiones son trasladables a poblaciones de diferentes características étnicas.

2. Barlow T. Early diagnosis and treatment of congenital dislocation of the hip. J Bone Joint Surg Br. 1962; 44: 829-848.

3. Godward S, Dezateux C. Surgery for congenital dislocation of the hip in the UK as a measure of outcome of screening. The Lancet.1998 April. 18:351:1149-1152.

4. RosendahL K, Markerstad T, Lie R.Congenital dislocation of the hip: a prospective study comparing ultrasound and clinical examination.Acta Pediatr.1992;1(2):177-81

5. Grover Geeta. Developmental hip dysplasia en Bercowitz ed. Pediatrics. A primary care approach. Saunders 1996. Cap 71 pg 296-299. 